\title{
Synthesis of enantiomeric spirooxazolines and spirooxazolidines by the regioselective ring closure of (-)- $\alpha$-pinene-based aminodiols
}

\author{
Zsolt Szakonyi, ${ }^{a}$ Anasztázia Hetényi, ${ }^{a}$ and Ferenc Fülöp ${ }^{\text {a,b* }}$ \\ ${ }^{a}$ Institute of Pharmaceutical Chemistry and ${ }^{b}$ Research Group of Stereochemistry of the \\ Hungarian Academy of Sciences, University of Szeged, H-6720 Szeged, Eötvös utca 6. Hungary \\ E-mail:fulop@pharm.u-szeged.hu
}

\section{Dedicated to Professor Csaba Szántay on the occasion of his $80^{\text {th }}$ birthday}

\begin{abstract}
Starting from $(1 R, 2 S, 3 S, 5 R)$-2-benzylaminomethyl-6,6-dimethylbicyclo[3.1.1]heptane-2,3-diol (4) and $(1 R, 2 S, 3 S, 5 R)$-2-aminomethyl-6,6-dimethylbicyclo[3.1.1]heptane-2,3-diol (5), spirooxazolines and spirooxazolidines were prepared. In the reactions of $\mathbf{4}$ and $\mathbf{5}$ with alkyl or aryl isothiocyanate, the ring closure proceeded regioselectively and resulted only in spiro derivatives of 2-aryl- and alkyliminooxazolidines 12-17. The primary aminodiol 5 was transformed with ethyl 4-chlorobenzimidate to a spiro-2-(4-chlorophenyl)oxazoline 18 in a highly regioselective ring closure. The reaction of $\mathbf{5}$ with formaldehyde resulted in tetracyclic compound 19.
\end{abstract}

Keywords: Monoterpene, chiral, aminodiol, regioselective reaction, oxazolidine, oxazoline

\section{Introduction}

Aliphatic aminodiols play important roles in drug therapy and drug research. ${ }^{1-7}$ For example, some have been found to act as HIV protease inhibitors ${ }^{1-3}$ and still others have been shown to exert renin inhibitor activity. ${ }^{5,6}$ Aminodiols are also useful starting materials for the syntheses of oxazines or oxazolidines, depending upon which hydroxy group undergoes ring closure with the amino group. ${ }^{8}$ Since the resulting heterobicycles contain a free hydroxy group, further ring closure can yield more complex heterocyclic structures. Additionally, alicyclic aminodiols are potentially excellent starting points for the development of new ring-chain tautomeric systems. ${ }^{8}$ Further, enantiomeric oxazolines and 1,3-oxazines have proved to be extremely efficient ligands in the asymmetric catalysis of a wide range of enantioselective syntheses. ${ }^{9}$ Chiral phosphineoxazine ligands and phosphine-oxazolidine ligands were recently successfully used in palladiumcatalyzed asymmetric allylic alkylation. ${ }^{10-13}$ 
Besides the chemical interest, several of these heterocyclic aminodiol systems exhibit noteworthy biological activity, e.g. cytoxazone, a selective modulator of $\mathrm{T}_{\mathrm{H}} 2$ cytokine secretion, which is a microbial metabolite isolated from Streptomyces species. ${ }^{14,15}$

As a continuation of our research on monoterpene-based $\beta$-amino acid derivatives, such as amino esters and amino alcohols, which have proved to be excellent building blocks for the syntheses of monoterpene-fused saturated 1,3-heterocycles, ${ }^{16-19}$ we recently reported syntheses of enantiomerically pure pinane-based aminodiol enantiomers, which were applied as chiral auxiliaries in enantioselective reactions of diethyl zinc with aromatic aldehydes. ${ }^{20}$ In the present work, our aim was to study the regioselectivity of the ring closure of aminodiols readily available in 4 steps from (-)- $\alpha$-pinene.

\section{Results and Discussion}

The starting enantiomeric aminodiols $\mathbf{4}$ and $\mathbf{5}$ were synthesized stereoselectively by a method described earlier. Starting from commercially available (-)- $\alpha$-pinene 1, epoxidation was followed by rearrangement of the $\alpha$-pinene oxide to trans-pinocarveol 2 , which was converted with MCPBA to epoxyalcohol 3 in a stereospecific reaction. ${ }^{21-23}$ Secondary aminodiol 4 was obtained by the reaction of $\mathbf{3}$ with benzylamine. Primary aminodiol $\mathbf{5}$ was prepared from $\mathbf{4}$ via debenzylation under standard conditions, by hydrogenation in the presence of palladium-oncarbon catalyst (Scheme 1). ${ }^{20}$

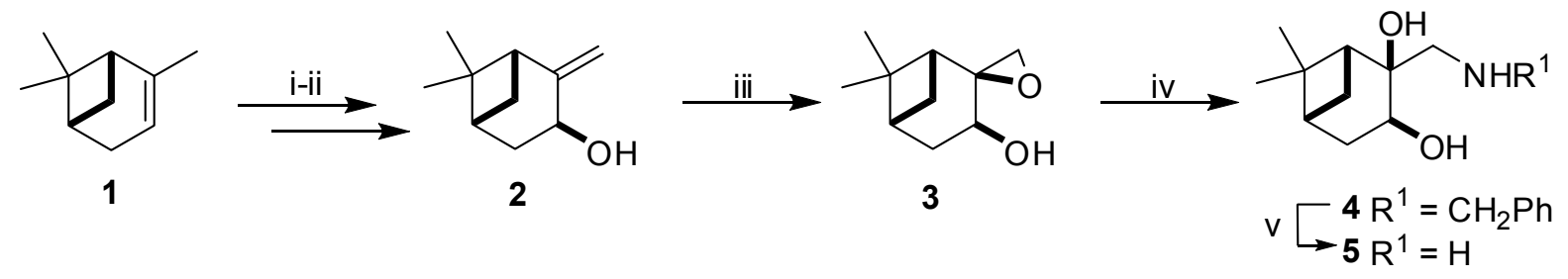

Scheme 1. (i) MCPBA, DCM, rt, 6 h, 82\%; (ii) $\mathrm{Al}(\mathrm{O} i \mathrm{Pr})_{3}$, toluene, reflux, $2 \mathrm{~h}, 70 \%$; (iii) MCPBA, DCM, $\mathrm{Na}_{2} \mathrm{HPO}_{4}$ buffer, rt, 12 h, 60\%; (iv) 4 equiv. $\mathrm{PhCH}_{2} \mathrm{NH}_{2}, 1$ equiv. $\mathrm{LiClO}_{4}$, $\mathrm{MeOH}$, reflux 3 days 63\%; (v) 10\% $\mathrm{Pd} / \mathrm{C}, \mathrm{MeOH}, \mathrm{H}_{2}, 1$ atm, 95\%.

In order to assess the tendencies of aminodiols $\mathbf{4}$ and $\mathbf{5}$ to furnish either a monoterpene spirooxazolidine or a monoterpene-condensed 1,3-oxazine ring, 4 and $\mathbf{5}$ were treated with phenyl, $m$-methoxyphenyl and ethyl isothiocyanates, which afforded the corresponding thiourea derivatives 6-11. After reaction with methyl iodide followed by treatment with base (KOH), these adducts underwent ring closure via methyl mercaptan elimination., ${ }^{8,24}$ The spirooxazolidine derivatives 12-17 were readily identified as the sole products in each respective case (Scheme 2). 


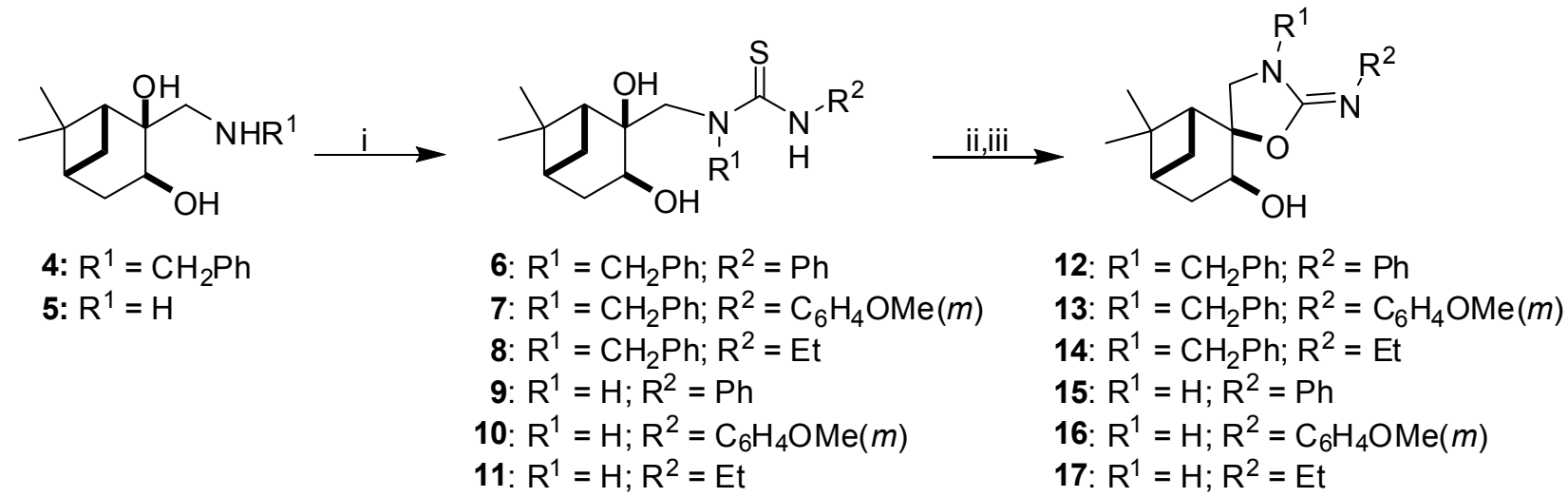

Scheme 2. (i) 1.05 equiv. $\mathrm{R}^{2} \mathrm{NCS}$, toluene, rt, $1-3 \mathrm{~h}, 75-90 \%$; (ii) 5.3 equiv. MeI, $\mathrm{MeOH}, 6$ h, rt; (iii) $2.5 \mathrm{M} \mathrm{KOH} / \mathrm{MeOH}, \mathrm{rt}, 4 \mathrm{~h}, 65-90 \%$.

For testing, the most stable structures formed for $\mathbf{1 2}$ and $\mathbf{1 5}$ were geometry optimized for both a monoterpene spirooxazolidine and a monoterpene-condensed 1,3-oxazine ring. Final minimizations were carried out by using Density functional theory (DFT) quantum mechanical method at the B3LYP/6-31G* level in vacuum. ${ }^{25}$ The DFT structures converged to the corresponding local minimum of the potential energy surface. The isomeric energy differences between the (-)- $\alpha$-pinene derived spirooxazolidines (e.g. 12 and 15) and the corresponding 1,3oxazines (e.g. 18 and 19) proved that oxazolidines are more stable than 1,3-oxazines, as shown in Figure 1.
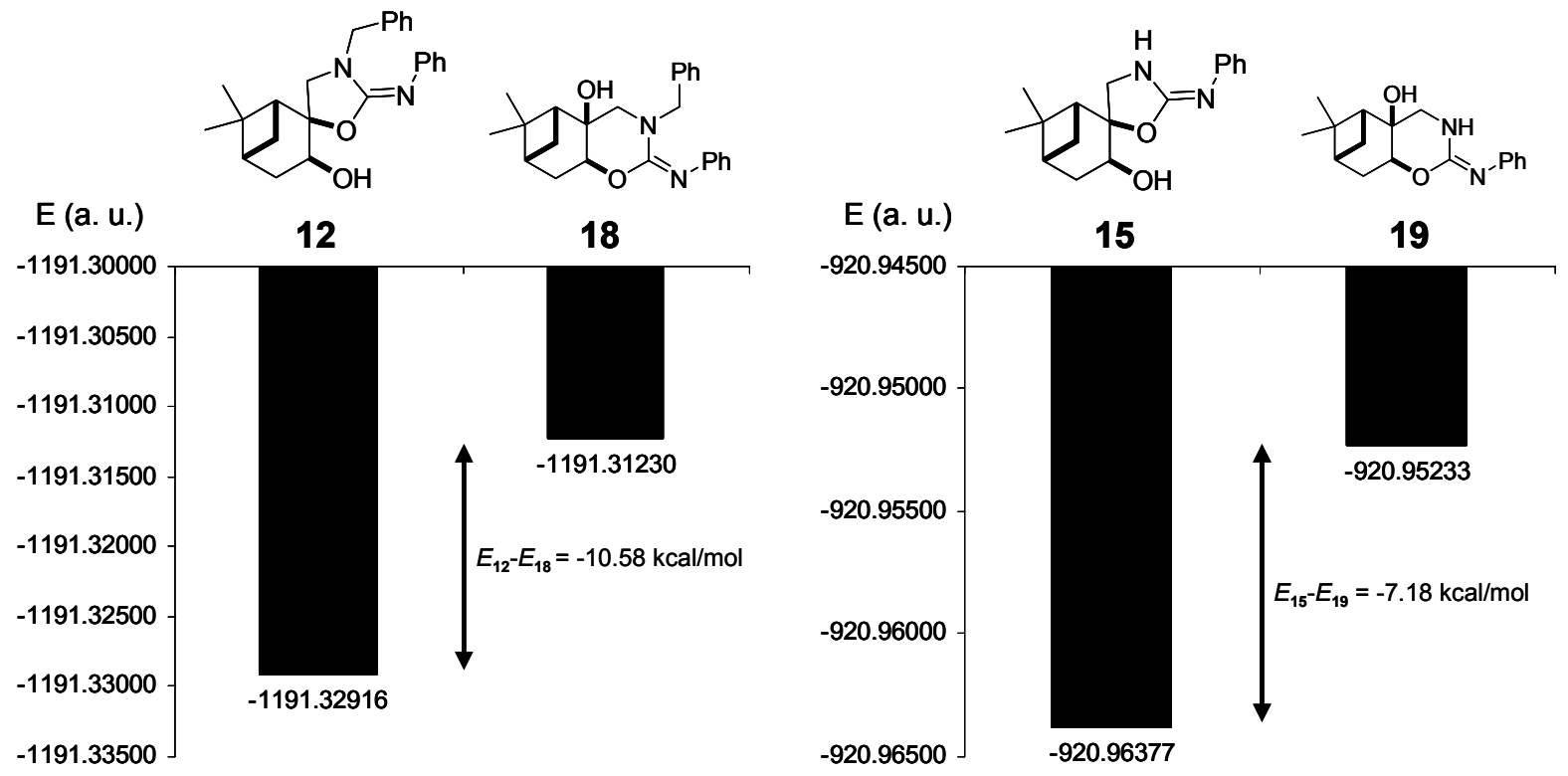

Figure 1. The isomeric energy difference between the spirooxazolidines (12 and 15) and 1,3oxazines (18 and 19); $E_{12}-E_{18}=-10.58 \mathrm{kcal} / \mathrm{mol} ; E_{15}-E_{19}=-7.18 \mathrm{kcal} / \mathrm{mol}$. 
The spirooxazolidine derivatives 12-17 and 20 were also distinguished from the 1,3-oxazines by the characteristic ${ }^{13} \mathrm{C}$ chemical shifts of C-3, C-4' and C-5' and by $2 \mathrm{D}$ heteronuclear NMR experiments. In the HMBC spectra, correlations between the C-3 proton and C-2' were not observed, implying that the products of the ring closure of thiourea derivatives 6-11 were spirooxazolidines. ${ }^{8}$

Another typical reaction of 1,2- and 1,3-aminoalcohols is their ring closure with imidates, resulting in 2-aryl- or 2-alkyl-substituted oxazolines or dihydro-1,3-oxazines. These 1,3heterocycles are catalysts often applied in enantioselective transformations, e.g. in palladiumcatalyzed asymmetric allylic alkylations. Starting from primary aminodiol 5, the ring closure with ethyl 4-chlorobenzimidate resulted in spirooxazoline $\mathbf{2 0}$ in a regioselective reaction (Scheme 3).

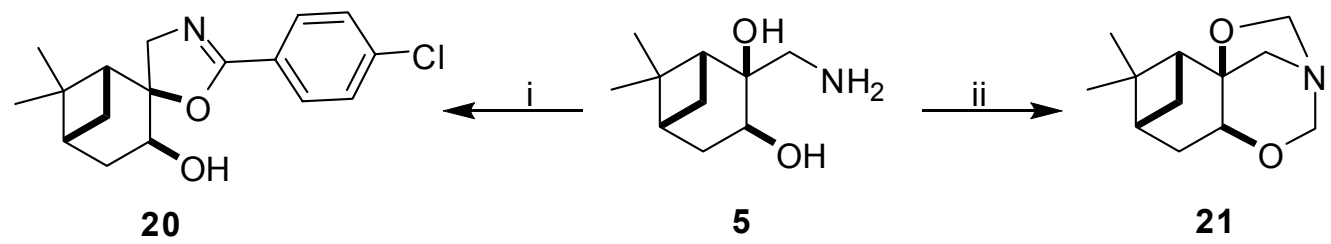

Scheme 3. (i) 4- $\mathrm{ClC}_{6} \mathrm{H}_{4} \mathrm{C}(=\mathrm{NH}) \mathrm{OEt}$, EtOH, cat. $\mathrm{AcOH}$, reflux, 8 h, 55\%; (ii) $\mathrm{CH}_{2} \mathrm{O} / \mathrm{H}_{2} \mathrm{O}, 4$ h, rt, $60 \%$.

Since the former reactions clearly demonstrated that formation of the spiro compound is preferable relative to the fused system, we applied formaldehyde for the ring closure to lead the secondary hydroxy group into a ring closure procedure. When aminodiol 5 was treated with formaldehyde solution, tetracyclic compound $\mathbf{2 1}$ was obtained. The first step is probably formation of the corresponding spiro compound, followed by conversation to 1,3-oxazine 21 .

\section{Experimental Section}

General Procedures. ${ }^{1} \mathrm{H}$ NMR spectra were recorded on a Bruker Avance DRX 400 spectrometer $\left(400 \mathrm{MHz}, \delta=0\right.$ (TMS), in $\mathrm{CDCl}_{3}$ or in DMSO-d6. Chemical shifts are expressed in ppm $(\delta)$ relative to TMS as internal reference. $J$ values are given in Hz. FT-IR spectra were recorded on an AVATAR 330 FT-IR spectrometer (Thermo Nicolet, USA). Microanalyses were performed on a Perkin-Elmer 2400 elemental analyzer.

GC measurements were made on a Perkin-Elmer Autosystem XL GC, consisting of a Flame Ionization Detector and a Turbochrom Workstation data system (Perkin-Elmer Corporation, Norwalk, USA). The column used for the direct separation of enantiomers was a CHIRASILDEX CB column (2500x0.25 mm I.D.). Optical rotations were obtained with a Perkin-Elmer 341 polarimeter. Melting points were determined on a Kofler apparatus and are uncorrected. The 
preparations of aminodiols 4 and $\mathbf{5}$ from $(1 S, 5 S)-(-)$ - $\alpha$-pinene were performed by a literature method. $^{20}$

\section{General procedure for the preparation of thiourea derivatives 6-11}

Ethyl, phenyl or 3-methoxyphenyl isothiocyanate $(1.70 \mathrm{mmol})$ was added to a solution of the appropriate aminodiol 4 or $5(1.62 \mathrm{mmol})$ in toluene $(15 \mathrm{~mL})$, and the resulting solution was stirred at room temperature. When the reaction was complete, as indicated by TLC (1-3 h), the solvent was removed under reduced pressure. The resulting crude crystalline products were recrystallized from EtOAc- $i \operatorname{Pr}_{2} \mathrm{O}$, while oily products were purified by column chromatography on silica gel with a toluene: $n$-hexane $=9: 1$ mixture.

\section{1-Benzyl-1-[(2,3-dihydroxy-6,6-dimethylbicyclo[3.1.1]hept-2-yl)methyl]-3-phenylthiourea}

(6). Yield: 88\%; m.p.: 147-149 ${ }^{\circ} \mathrm{C} ; \quad[\alpha]^{20}{ }_{\mathrm{D}}+107 \quad(0.25$, EtOH); Anal. Calcd for $\mathrm{C}_{24} \mathrm{H}_{30} \mathrm{~N}_{2} \mathrm{O}_{2} \mathrm{~S}$ (410.57): C, 70.21; H, 7.36; N, 6.82\%. Found: C, 70.46; H, 7.88; N, 6.53\%. ${ }^{1} \mathrm{H}$ NMR $\left(\mathrm{CDCl}_{3}\right) \delta(\mathrm{ppm}): 0.93(3 \mathrm{H}, s), 1.31(3 \mathrm{H}, s), 1.46(1 \mathrm{H}, d, J=10.2 \mathrm{~Hz}), 1.69(1 \mathrm{H}, d d d$, $J=2.4,5.1,14.1 \mathrm{~Hz}), 1.94-1.98(1 \mathrm{H}, m), 2.23-2.31(1 \mathrm{H}, m), 2.46-2.52(1 \mathrm{H}, m), 3.64(1 \mathrm{H}, d, J=$ $15.4 \mathrm{~Hz}), 4.03-4.11(1 \mathrm{H}, m), 4.25(1 \mathrm{H}, d d, J=5.3,9.2 \mathrm{~Hz}), 4.90(1 \mathrm{H}, b r s), 5.15(1 \mathrm{H}, d, J=15.6$ $\mathrm{Hz}), 5.40(1 \mathrm{H}, d, J=15.4 \mathrm{~Hz}), 7.15-7.18(1 \mathrm{H}, m), 7.30-7.42(9 \mathrm{H}, m), 9.30(1 \mathrm{H}, b r s) .{ }^{13} \mathrm{C}$ NMR $\left(\mathrm{CDCl}_{3}\right) \delta(\mathrm{ppm}):$ 24.1, 27.6, 27.9, 37.7, 38.7, 40.5, 51.0, 57.2, 66.0, 77.8, 124.8, 125.3, 126.9, $127.8,128.5,129.0,140.2,185.4$.

\section{1-Benzyl-1-[(2,3-dihydroxy-6,6-dimethylbicyclo[3.1.1]hept-2-yl)methyl]-3-(3'-methoxy-} phenyl)thiourea (7). Yield: $77 \%$; m.p.: $149-151^{\circ} \mathrm{C}$; $[\alpha]^{20}{ }_{\mathrm{D}}+82(0.25$, EtOH); Anal. Calcd for $\mathrm{C}_{25} \mathrm{H}_{32} \mathrm{~N}_{2} \mathrm{O}_{3} \mathrm{~S}$ (440.60): C, 68.15; H, 7.32; N, 6.36\%. Found: C, 68.43; H, 7.54; N, 6.11\%; ${ }^{1} \mathrm{H}$ $\operatorname{NMR}\left(\mathrm{CDCl}_{3}\right) \delta(\mathrm{ppm}): 0.87(3 \mathrm{H}, s), 1.30(3 \mathrm{H}, s), 1.40(1 \mathrm{H}, d, J=9.7 \mathrm{~Hz}), 1.66(1 \mathrm{H}, d d d, J=$ 2.0, 4.8, $14.2 \mathrm{~Hz}), 1.91-1.95(1 \mathrm{H}, m), 2.23-2.29(2 \mathrm{H}, m), 2.42-2.48(1 \mathrm{H}, m), 3.46-3.54(2 \mathrm{H}, m)$, $3.79(1 \mathrm{H}, s), 3.97(1 \mathrm{H}, b r s), 4.10(1 \mathrm{H}, b r s), 5.06(1 \mathrm{H}, b r s), 5.44(1 \mathrm{H}, b r s), 6.70(1 \mathrm{H}, d d, J=$ 2.2, 8.2 Hz), $6.94(1 \mathrm{H}, d, J=7.4 \mathrm{~Hz}), 7.10(1 \mathrm{H}, s), 7.21(1 \mathrm{H}, t, J=8.1 \mathrm{~Hz}), 7.28-7.34(3 \mathrm{H}, m)$, $7.39(2 \mathrm{H}, t, J=7.6 \mathrm{~Hz}), 9.80(1 \mathrm{H}, b r s) .{ }^{13} \mathrm{C} \mathrm{NMR}\left(\mathrm{CDCl}_{3}\right) \delta(\mathrm{ppm}): 24.0,27.5,27.8,37.7,38.6$, $40.3,50.2$, 55.4, 57.1, 60.2, 65.6, 77.9, 110.4, 110.9, 116.9, 127.0, 127.7, 128.9, 129.2, 135.9, $141.4,159.8,185.0$.

1-Benzyl-1-[(2,3-dihydroxy-6,6-dimethylbicyclo[3.1.1]hept-2-yl)methyl]-3-ethylthiourea (8). Yield: 75\%; an oil; $[\alpha]^{20}{ }_{\mathrm{D}}$ : $+50\left(0.28\right.$, EtOH); Anal. Calcd for $\mathrm{C}_{20} \mathrm{H}_{30} \mathrm{~N}_{2} \mathrm{O}_{2} \mathrm{~S}$ (362.53): C, 66.26; H, 8.34; N, 7.73\%. Found: C, 66.51; H, 8.02; N, 7.96\%; ${ }^{1} \mathrm{H}$ NMR $\left(\mathrm{CDCl}_{3}\right) \delta$ (ppm): $0.67(3 \mathrm{H}, s)$, $0.92(1 \mathrm{H}, t, J=7.3 \mathrm{~Hz}), 1.05(3 \mathrm{H}, s), 1.19(1 \mathrm{H}, d, J=10.3 \mathrm{~Hz}), 1.46(1 \mathrm{H}, d d d, J=2.3,5.2,14.2$ $\mathrm{Hz}), 1.68-1.72(1 \mathrm{H}, m), 1.96(1 \mathrm{H}, t, J=5.9 \mathrm{~Hz}), 1.99-2.04(1 \mathrm{H}, m), 2.21-2.27(1 \mathrm{H}, m), 3.21(1 \mathrm{H}$, $d, J=15.3 \mathrm{~Hz}), 3.36-3.41(2 \mathrm{H}, m), 3.80(1 \mathrm{H}$, br s $), 3.98(1 \mathrm{H}, d d, J=5.0,9.0 \mathrm{~Hz}), 4.89(1 \mathrm{H}$, br s$)$, $6.99(1 \mathrm{H}, d, J=7.6 \mathrm{~Hz}), 7.04-7.07(1 \mathrm{H}, m), 7.12(1 \mathrm{H}, t, J=7.5 \mathrm{~Hz}) .{ }^{13} \mathrm{C} \mathrm{NMR}\left(\mathrm{CDCl}_{3}\right) \delta(\mathrm{ppm})$ : $14.3,24.1,27.6,27.8,37.8,38.6,40.4,41.4,50.6,56.6,60.3,65.6,77.6,126.7,127.6,128.9$, 135.8, 184.6.

1-[(2,3-Dihydroxy-6,6-dimethylbicyclo[3.1.1]hept-2-yl)methyl]-3-phenylthiourea (9). Yield: 90\%; m.p.: $110-113{ }^{\circ} \mathrm{C}$; $[\alpha]^{20}{ }_{\mathrm{D}}:-12$ (0.25, EtOH); Anal. Calcd for $\mathrm{C}_{17} \mathrm{H}_{24} \mathrm{~N}_{2} \mathrm{O}_{2} \mathrm{~S}(320.45)$ : C, 
63.72; H, 7.55; N, 8.74\%. Found: C, 64.11; H, 7.89; N, 8.36\%. ${ }^{1} \mathrm{H}$ NMR $\left(\mathrm{CDCl}_{3}\right) \delta(\mathrm{ppm}): 1.04$ $(3 \mathrm{H}, s), 1.26(3 \mathrm{H}, s), 1.37(1 \mathrm{H}, d, J=10.4 \mathrm{~Hz}), 1.63(1 \mathrm{H}, d d d, \mathrm{~J}=2.3,4.6,14.1 \mathrm{~Hz}), 1.89-1.93$ $(1 \mathrm{H}, m), 2.02-2.05(1 \mathrm{H}, m), 2.18-2.23(1 \mathrm{H}, m), 2.45-2.51(1 \mathrm{H}, m), 3.46(1 \mathrm{H}, b r s), 3.60(1 \mathrm{H}, b r$ $s), 3.99(1 \mathrm{H}, b r s), 4.12(1 \mathrm{H}, b r s), 4.20-4.27(1 \mathrm{H}, m), 6.86(1 \mathrm{H}, b r s), 7.22-7.30(3 \mathrm{H}, m), 7.36-$ $7.41(2 \mathrm{H}, m), 8.42(1 \mathrm{H}, b r s) .{ }^{13} \mathrm{C} \mathrm{NMR}\left(\mathrm{CDCl}_{3}\right) \delta(\mathrm{ppm}): 24.1,27.5,27.6,38.1,38.8,40.5,50.3$, $54.7,65.8,124.8,127.0,130.0,136.2,183.2$.

1-[(2,3-Dihydroxy-6,6-dimethylbicyclo[3.1.1]hept-2-yl)methyl]-3-(3'-methoxyphenyl)thiourea (10). Yield: 87\%; m.p.: 83-85 ${ }^{\circ} \mathrm{C} ;[\alpha]_{\mathrm{D}}^{20}:-11 \quad(0.25$, EtOH); Anal. Calcd for $\mathrm{C}_{18} \mathrm{H}_{26} \mathrm{~N}_{2} \mathrm{O}_{2} \mathrm{~S}$ (350.48): C, 61.69; H, 7.48; N, 7.99\%. Found: C, 61.85; H, 7.23; N, 8.12\%; ${ }^{1} \mathrm{H}$ NMR $\left(\mathrm{CDCl}_{3}\right) \delta(\mathrm{ppm}): 1.05(3 \mathrm{H}, s), 1.27(3 \mathrm{H}, s), 1.39(1 \mathrm{H}, d, J=10.4 \mathrm{~Hz}), 1.62-1.67(1 \mathrm{H}, m)$, 1.90-1.94 (1H, m), $2.05(1 \mathrm{H}, t, J=5.7 \mathrm{~Hz}), 2.18-2.24(1 \mathrm{H}, m), 2.49(1 \mathrm{H}, t, J=11.2 \mathrm{~Hz}), 3.28$ $(1 \mathrm{H}, b r s), 3.62(1 \mathrm{H}, d d, J=3.8,13.5 \mathrm{~Hz}), 3.79(3 \mathrm{H}, s), 3.98(1 \mathrm{H}, b r s), 4.23-4.28(1 \mathrm{H}, m), 6.79$ $(1 \mathrm{H}, d, J=8.3 \mathrm{~Hz}), 6.83-6.87(2 \mathrm{H}, m), 6.96(2 \mathrm{H}, b r s), 7.26-7.30(1 \mathrm{H}, m), 8.26(2 \mathrm{H}, b r s) .{ }^{13} \mathrm{C}$ NMR $\left(\mathrm{CDCl}_{3}\right) \delta(\mathrm{ppm}): 24.0,27.5,27.6,38.1,38.9,40.6,50.5,54.8,55.4,65.9,76.0,110.1$, $112.8,116.5,130.7,160.8,180.8$.

1-[(2,3-Dihydroxy-6,6-dimethylbicyclo[3.1.1]hept-2-yl)methyl]-3-ethylthiourea (11). Yield: 85\%; m.p.: $140-142{ }^{\circ} \mathrm{C}$; $[\alpha]^{20}{ }_{\mathrm{D}}$ : -8 (0.25, EtOH); Anal. Calcd for $\mathrm{C}_{13} \mathrm{H}_{24} \mathrm{~N}_{2} \mathrm{O}_{2} \mathrm{~S}(272.41)$ : C, 57.32; H, 8.88; N, 10.28\%. Found: C, 57.55; H, 8.63; N, 10.51\%; ${ }^{1} \mathrm{H} \mathrm{NMR}\left(\mathrm{CDCl}_{3}\right) \delta(\mathrm{ppm})$ : $1.02(3 \mathrm{H}, s), 1.23(1 \mathrm{H}, t, J=7.2 \mathrm{~Hz}), 1.29(3 \mathrm{H}, s), 1.42(1 \mathrm{H}, d, J=10.6 \mathrm{~Hz}), 1.68(1 \mathrm{H}, d d d, J=$ $2.2,4.8,14.1 \mathrm{~Hz}), 1.92-1.96(1 \mathrm{H}, m), 2.08(1 \mathrm{H}, t, J=5.7 \mathrm{~Hz}), 2.22-2.27(1 \mathrm{H}, m), 2.47-2.53(1 \mathrm{H}$, $m), 3.44-3.52(3 \mathrm{H}, m), 4.26(1 \mathrm{H}, d d, J=5.1,9.4 \mathrm{~Hz}), 6.80-6.94(2 \mathrm{H}, m) .{ }^{13} \mathrm{C} \mathrm{NMR}\left(\mathrm{CDCl}_{3}\right) \delta$ (ppm): 14.2, 24.1, 27.5, 27.6, 37.7, 37.8, 38.8, 40.6, 50.5, 54.5, 65.7, 76.4, 182.6.

General procedure for the preparation of spiro-2-iminooxazolidine derivatives 12-17 Iodomethane $(0.53 \mathrm{~g}, 3.70 \mathrm{mmol})$ was added dropwise to a solution of the appropriate thiourea adduct 6-11 $(0.70 \mathrm{mmol})$ in dry methanol $(10 \mathrm{~mL})$. After stirring for $6 \mathrm{~h}$ at room temperature, the solvent was removed under reduced pressure, after which a methanolic solution of $\mathrm{KOH}(2.5 \mathrm{M}$, $10 \mathrm{~mL}$ ) was added to the residue. The resulting solution was then stirred for $4 \mathrm{~h}$, followed by evaporation to dryness. Water $(30 \mathrm{~mL})$ was added to this residue and the resulting suspension was extracted with $\mathrm{CHCl}_{3}(3 \times 40 \mathrm{~mL})$. The organic layer was dried over $\mathrm{Na}_{2} \mathrm{SO}_{4}$, filtered and then evaporated to dryness, yielding in a crystalline product, which was purified by flash chromatography over silica gel with an EtOAc: $n$-hexane $=1: 1$ mixture.

\section{3'-Benzyl-6,6-dimethyl-2'-(phenylimino)spiro[bicyclo[3.1.1]heptane-2,5'-oxazolidin]-3-ol}

(12). Yield: 64\%; m.p.: $140-141^{\circ} \mathrm{C} ; \quad[\alpha]^{20}{ }_{\mathrm{D}}$; $3 \quad(0.25$, EtOH); Anal. Calcd for $\mathrm{C}_{24} \mathrm{H}_{28} \mathrm{~N}_{2} \mathrm{O}_{2}$ (376.49): C, 76.56; H, 7.50; N, 7.44\%. Found: C, 76.98; H, 7.54; N, 7.00\%; ${ }^{1} \mathrm{H}$ $\operatorname{NMR}\left(\mathrm{CDCl}_{3}\right) \delta(\mathrm{ppm}): 0.79(3 \mathrm{H}, s), 1.25(3 \mathrm{H}, s), 1.47(1 \mathrm{H}, d, J=10.4 \mathrm{~Hz}), 1.69-1.77(1 \mathrm{H}, m)$, 1.94-2.01 (1H, m), 2.22-2.33 (2H, m), 2.37-2.47 (1H, m), $3.18(1 \mathrm{H}, d, J=8.7 \mathrm{~Hz}), 3.34(1 \mathrm{H}, d, J$ $=8.7 \mathrm{~Hz}), 4.11(1 \mathrm{H}, d d, J=4.9,8.3 \mathrm{~Hz}), 4.52(1 \mathrm{H}, d, J=15.1 \mathrm{~Hz}), 4.66(1 \mathrm{H}, d, J=15.1 \mathrm{~Hz})$, $6.96(1 \mathrm{H}, t, J=7.2 \mathrm{~Hz}), 7,10-7,38(9 \mathrm{H}, m) .{ }^{13} \mathrm{C} \mathrm{NMR}\left(\mathrm{CDCl}_{3}\right) \delta(\mathrm{ppm}): 23.1,27.1,27.7,37.3$, $38.8,40.2,48.9,51.2,56.9,69.5,86.2,122.0,123.5,127.6,128.0,128.5,128.7,136.5,151.5$. 
3'-Benzyl-6,6-dimethyl-2'-[(3-methoxyphenyl)imino]spiro[bicyclo[3.1.1]heptane-2,5'oxazolidin]-3-ol (13). Yield: 88\%; m.p.: 116-118 ${ }^{\circ} \mathrm{C}$; $[\alpha]^{20}{ }_{\mathrm{D}}:+36(0.25$, EtOH); Anal. Calcd for $\mathrm{C}_{25} \mathrm{H}_{30} \mathrm{~N}_{2} \mathrm{O}_{3}$ (406.52): C, 73.86; H, 7.44; N, 6.89\%. Found: C, 73.97; H, 7.31; N, 6.99\%; ${ }^{1} \mathrm{H}$ $\operatorname{NMR}\left(\mathrm{CDCl}_{3}\right) \delta(\mathrm{ppm}): 0.63(3 \mathrm{H}, s), 1.08(3 \mathrm{H}, s), 1.32(1 \mathrm{H}, d, J=10.5 \mathrm{~Hz}), 1.53-1.59(1 \mathrm{H}, m)$, 1.79-1.82 (1H, $m), 2.09(1 \mathrm{H}, t, J=5.8 \mathrm{~Hz}), 2.11-2.15(1 \mathrm{H}, m), 2.22-2.30(2 \mathrm{H}, m), 3.01(1 \mathrm{H}, d, J$ $=8.7 \mathrm{~Hz}), 3.15(1 \mathrm{H}, d, J=8.7 \mathrm{~Hz}), 3.60(3 \mathrm{H}, s), 3.90-3.95(1 \mathrm{H}, m), 4.35(1 \mathrm{H}, d, J=15.4 \mathrm{~Hz})$, $4.42(1 \mathrm{H}, d, J=15.4 \mathrm{~Hz}), 6.37(1 \mathrm{H}, d d, J=2.1,8.3 \mathrm{~Hz}), 6.52-6.55(2 \mathrm{H}, m), 6.96(1 \mathrm{H}, t, J=8.1$ $\mathrm{Hz}), 7.10-7.20(5 \mathrm{H}, m) .{ }^{13} \mathrm{C} \mathrm{NMR}\left(\mathrm{CDCl}_{3}\right) \delta(\mathrm{ppm}): 23.1,27.2,27.8,37.5,38.8,40.3,48.9,51.3$, 55.2, 56.8, 69.5, 85.9, 108.1, 109.0, 116.1, 127.6, 128.0, 128.7, 129.1, 136.7, 149.0, 151.4, 160.0. 3'-Benzyl-2'-(ethylimino)-6,6-dimethylspiro[bicyclo[3.1.1] heptane-2,5'-oxazolidin]-3-ol (14). Yield: 66\%; an oil; $[\alpha]^{20}{ }_{\mathrm{D}}$ : +2 (0.30, EtOH); Anal. Calcd for $\mathrm{C}_{20} \mathrm{H}_{28} \mathrm{~N}_{2} \mathrm{O}_{2}$ (328.45): C, 73.14; $\mathrm{H}$, 8.59; N, 8.53\%. Found: C, 73.42; H, 8.65; N, 8.21\%; ${ }^{1} \mathrm{H} \mathrm{NMR}\left(\mathrm{CDCl}_{3}\right) \delta(\mathrm{ppm}): 0.77(3 \mathrm{H}, s)$, $1.15(1 \mathrm{H}, t, J=7.2 \mathrm{~Hz}), 1.24(3 \mathrm{H}, s), 1.56(1 \mathrm{H}, d, J=10.7 \mathrm{~Hz}), 1.80(1 \mathrm{H}, d d d, J=3.0,4.4,14.2$ $\mathrm{Hz}), 1.96-2.00(1 \mathrm{H}, m), 2.19(1 \mathrm{H}, t, J=5.6 \mathrm{~Hz}), 2.25-2.30(1 \mathrm{H}, m), 2.39-2.44(1 \mathrm{H}, m), 3.10(1 \mathrm{H}$, $d, J=8.6 \mathrm{~Hz}), 3.21(1 \mathrm{H}, d, J=8.6 \mathrm{~Hz}), 3.30(2 \mathrm{H}, q, J=7.0 \mathrm{~Hz}), 4.07(1 \mathrm{H}, d d, J=4.6,8.9 \mathrm{~Hz})$, $4.35(1 \mathrm{H}, d, J=15.1 \mathrm{~Hz}), 4.42(1 \mathrm{H}, d, J=15.1 \mathrm{~Hz}), 7.24-7.27(3 \mathrm{H}, m), 7.30-7.33(2 \mathrm{H}, m) .{ }^{13} \mathrm{C}$ $\operatorname{NMR}\left(\mathrm{CDCl}_{3}\right) \delta$ (ppm): 17.1, 23.1, 27.2, 27.4, 37.3, 38.9, 40.2, 41.0, 49.0, 51.0, 57.5, 59.1, 84.5, $127.3,127.9,128.6,137.1,152.4$.

6,6-Dimethyl-2'-(phenylimino)spiro[bicyclo[3.1.1]heptane-2,5'-oxazolidin]-3-ol (15). This compound was purified as a hydrochloride salt. Yield: $65 \%$; m.p.: $175-178{ }^{\circ} \mathrm{C} ;[\alpha]^{20}{ }_{\mathrm{D}}:-1.0$ (0.25, EtOH); Anal. Calcd for $\mathrm{C}_{17} \mathrm{H}_{23} \mathrm{ClN}_{2} \mathrm{O}_{2}$ (322.83): C, 63.25; H, 7.18; N 8.68\%. Found: C, 63.37; H, 7.28; N, 8.29\%; ${ }^{1} \mathrm{H}$ NMR (DMSO-d6) $\delta$ (ppm): $0.88(3 \mathrm{H}, s), 1.24(3 \mathrm{H}, s), 1.47(1 \mathrm{H}, d$, $J=9.9 \mathrm{~Hz}), 1.66(1 \mathrm{H}, d d d, J=2.9,4.8,13.9 \mathrm{~Hz}), 1.89-1.96(1 \mathrm{H}, m), 2.10(1 \mathrm{H}, t, J=5.5 \mathrm{~Hz})$, 2.19-2.27 (1H, $m), 2.29-2.38(1 \mathrm{H}, m), 3.57(1 \mathrm{H}, d, J=12.1 \mathrm{~Hz}), 3.66(1 \mathrm{H}, d, J=12.1 \mathrm{~Hz}), 4,04-$ 4.,12 (1H, $m), 4,87(1 \mathrm{H}, d, J=5.5 \mathrm{~Hz}), 6.84(1 \mathrm{H}, t, J=7.3 \mathrm{~Hz}), 7.18(2 \mathrm{H}, t, J=7.8 \mathrm{~Hz}), 7.46-$ $7.60(2 \mathrm{H}, m), 8.90(1 \mathrm{H}, b r s) .{ }^{13} \mathrm{C} \mathrm{NMR}\left(\mathrm{CDCl}_{3}\right) \delta(\mathrm{ppm}): 23.1,27.0,27.9,37.2,38.1,39.2,39.8$, $51.4,69.4,88.0,117.5,120.4,128.3,155.0,171.0$.

\section{6,6-Dimethyl-2'-[(3-methoxyphenyl)imino]spiro[bicyclo[3.1.1]heptane-2,5'-oxazolidin]-3-ol}

(16). Yield: 90\%; m.p.: $143-145^{\circ} \mathrm{C} ; \quad[\alpha]^{20}{ }_{\mathrm{D}}$ : $+11 \quad(0.25$, EtOH); Anal. Calcd for $\mathrm{C}_{17} \mathrm{H}_{22} \mathrm{~N}_{2} \mathrm{O}_{2}$ (286.37): C, 71.30; H, 7.74; N, 9.78\%. Found: C, 71.45; H, 7.54; N, 9.93\%; ${ }^{1} \mathrm{H}$ NMR $\left(\mathrm{CDCl}_{3}\right) \delta(\mathrm{ppm}): 0.89(3 \mathrm{H}, s), 1.31(3 \mathrm{H}, s), 1.55(1 \mathrm{H}, d, J=8.8 \mathrm{~Hz}), 1.80-1.84(1 \mathrm{H}, m)$, 1.99-2.03 $(1 \mathrm{H}, m), 1.99-2.03(1 \mathrm{H}, m), 1.99-2.03(1 \mathrm{H}, m), 2.28-2.33(2 \mathrm{H}, m), 2.43-2.49(1 \mathrm{H}, m)$, 3.63-3.79 $(5 \mathrm{H}, m), 4.18(1 \mathrm{H}, d d, J=4.6,8.8 \mathrm{~Hz}), 6.55(1 \mathrm{H}, d d, J=1.8,8.3 \mathrm{~Hz}), 6.84(1 \mathrm{H}, b r s)$, $7.00(1 \mathrm{H}, b r s), 7.15(1 \mathrm{H}, t, J=8.3 \mathrm{~Hz}) .{ }^{13} \mathrm{C} \mathrm{NMR}\left(\mathrm{CDCl}_{3}\right) \delta(\mathrm{ppm}): 23.2,27.1,28.0,37.3,38.8$, $39.5,40.4,51.5,55.2,70.4,89.9,108.2,129.6,141.3,160.2,160.4$.

2'-(Ethylimino)-6,6-dimethylspiro[bicyclo[3.1.1] heptane-2,5'-oxazolidin]-3-ol (17). Yield: 77\%; m.p.: $131-133{ }^{\circ} \mathrm{C}$; $[\alpha]^{20}{ }_{\mathrm{D}}:+5$ (0.25, EtOH); Anal. Calcd for $\mathrm{C}_{13} \mathrm{H}_{22} \mathrm{~N}_{2} \mathrm{O}_{2}$ (238.33): C, 65.51; H, 9.30; N, 11.75\%. Found: C, 65.75; H, 9.21; N, 11.83\%; ${ }^{1} \mathrm{H} \mathrm{NMR}\left(\mathrm{CDCl}_{3}\right) \delta(\mathrm{ppm})$ : $0.84(3 \mathrm{H}, s), 1.13(3 \mathrm{H}, \mathrm{t}, J=7.2 \mathrm{~Hz}), 1.24(3 \mathrm{H}, s), 1.45(1 \mathrm{H}, d, J=10.2 \mathrm{~Hz}), 1.74-1.79(1 \mathrm{H}, m)$, 1.93-1.96 $(1 \mathrm{H}, m), 2.18(3 \mathrm{H}, \mathrm{t}, J=5.4 \mathrm{~Hz}), 2.20-2.25(1 \mathrm{H}, m), 2.36-2.42(1 \mathrm{H}, m), 3.18(1 \mathrm{H}, d, J$ 
$=7.0 \mathrm{~Hz}), 3.20(1 \mathrm{H}, d, J=7.0 \mathrm{~Hz}), 3.58(1 \mathrm{H}, d, J=12.3 \mathrm{~Hz}), 3.64(1 \mathrm{H}, d, J=7.0 \mathrm{~Hz}), 4.10$ $(1 \mathrm{H}, d d, J=4.2,8.8 \mathrm{~Hz}) .{ }^{13} \mathrm{C} \mathrm{NMR}\left(\mathrm{CDCl}_{3}\right) \delta(\mathrm{ppm}): 15.1,23.2,27.1,27.7,37.2,37.6,38.8$, $40.3,51.6,66.3,70.5,90.2,158.8$.

\section{6,6-Dimethyl-2'-(4-chlorophenyl)-4' $H$-spiro[bicyclo[3.1.1] heptane-2,5'-oxazol]-3-ol}

Ethyl 4-chlorobenzimidate $(0.18 \mathrm{~g}, 1 \mathrm{mmol})$ and 1 drop of glacial acetic acid were added to a solution of aminodiol $5(0.19 \mathrm{~g}, 1 \mathrm{mmol})$ in $15 \mathrm{~mL}$ of dry ethanol. The solution was refluxed for $8 \mathrm{~h}$, and then evaporated, and the crude product was purified by flash chromatography over silica gel with an EtOAc: $n$-hexane $=2: 1$ mixture $\left(R_{\mathrm{f}}=0.24\right)$. Yield: $0.17 \mathrm{~g}(55 \%)$; m.p.: $146-147^{\circ} \mathrm{C}$; $[\alpha]^{20}{ }_{\mathrm{D}}$ : -2 (0.25, EtOH); Anal. Calcd for $\mathrm{C}_{17} \mathrm{H}_{20} \mathrm{ClNO}_{2}$ (305.80): C, 66.77; H, 6.59; N, 4.58\%. Found: C, 66.83; H, 6.87; N, 4.90\%; ${ }^{1} \mathrm{H}$ NMR $\left(\mathrm{CDCl}_{3}\right) \delta(\mathrm{ppm}): 0.94(3 \mathrm{H}, s), 1.31(3 \mathrm{H}, s), 1.64$ $(1 \mathrm{H}, d, J=10.5 \mathrm{~Hz}), 1.87(1 \mathrm{H}, d t, J=3.6,14.3 \mathrm{~Hz}), 2.02-2.08(1 \mathrm{H}, m), 2.24(1 \mathrm{H}, t, J=5.6 \mathrm{~Hz})$, 2.30-2.38 $(1 \mathrm{H}, m), 2.46-2.54(2 \mathrm{H}, m), 3.75(1 \mathrm{H}, d, J=15.1 \mathrm{~Hz}), 3.84(1 \mathrm{H}, d, J=15.1 \mathrm{~Hz}), 4.15-$ $4.21(1 \mathrm{H}, m), 7.39(2 \mathrm{H}, d, J=8.5 \mathrm{~Hz}), 7.87(2 \mathrm{H}, d, J=8.5 \mathrm{~Hz}) .{ }^{13} \mathrm{C} \mathrm{NMR}\left(\mathrm{CDCl}_{3}\right) \delta(\mathrm{ppm})$ : 23.6, 27.4, 28.5, 37.9, 38.6, 40.4, 52.4, 68.3, 70.8, 90.6, 127.5, 129.0, 130.2, 136.3, 161.3.

\section{3,3-Dimethyl-2,4-methylene-7,11-dioxa-9-azatricyclo[7.2.1.0 $\left.{ }^{1,6}\right]$ dodecane (21)}

Aminodiol 5 was stirred with $10 \mathrm{~mL}$ of $33 \%$ aqueous formaldehyde for $4 \mathrm{~h}$ at room temperature. Water $(30 \mathrm{~mL})$ was next added to the reaction mixture and the solution was extracted with $\mathrm{CHCl}_{3}(3 \times 40 \mathrm{~mL})$. The organic layer was dried over $\mathrm{Na}_{2} \mathrm{SO}_{4}$, filtered and then evaporated to dryness, furnishing in a colorless oil, which was purified by flash chromatography over silica gel with a toluene:EtOH $=9: 1$ mixture $\left(R_{\mathrm{f}}=0.35\right)$. Yield: $60 \%$; a colorless oil; $[\alpha]^{20}{ }_{\mathrm{D}}:+7(0.30$, EtOH); Anal. Calcd for $\mathrm{C}_{12} \mathrm{H}_{19} \mathrm{NO}_{2}$ (209.28): C, 68.87; H, 9.15; N, 6.69\%. Found: C, 69.09; H, 9.31; N, 6.78\%; ${ }^{1} \mathrm{H}$ NMR $\left(\mathrm{CDCl}_{3}\right) \delta(\mathrm{ppm}): 1.07(3 \mathrm{H}, s), 1.29(3 \mathrm{H}, s), 1.71(1 \mathrm{H}, d d, J=9.8,13.2$ $\mathrm{Hz}), 1.77(1 \mathrm{H}, d, J=10.0 \mathrm{~Hz}), 1.97(1 \mathrm{H}, q, J=5.9 \mathrm{~Hz}), 2.04(1 \mathrm{H}, t, J=5.8 \mathrm{~Hz}), 2.14-2.17(1 \mathrm{H}$, $m), 2.33-2.35(1 \mathrm{H}, m), 2.79(1 \mathrm{H}, d, J=11.2 \mathrm{~Hz}), 3.26(1 \mathrm{H}, d, J=11.2 \mathrm{~Hz}), 4.17(1 \mathrm{H}, t, J=9.0$ $\mathrm{Hz}), 4.37(1 \mathrm{H}, d d, J=1.5,5.3 \mathrm{~Hz}), 4.41(1 \mathrm{H}, d d, J=1.4,9.8 \mathrm{~Hz}), 4.51(1 \mathrm{H}, d d, J=1.1,9.8 \mathrm{~Hz})$, $4.70(1 \mathrm{H}, d, J=5.3 \mathrm{~Hz}) .{ }^{13} \mathrm{C} \mathrm{NMR}\left(\mathrm{CDCl}_{3}\right) \delta(\mathrm{ppm}): 24.5,29.1,31.9,32.0,39.0,41.8,48.8$, $62.7,77.5,81.0,83.3,87.3$.

\section{Acknowledgements}

We are grateful to the Hungarian Research Foundation (OTKA No. NF69316 and T049407) and the National Research and Development Office, Hungary (GVOP-311-2004-05-0255/3.0) for financial support. We thank R. Kovács for assistance in the experimental work. 


\section{References and Notes}

1. Kempf, D. J.; Sowin, T. J.; Doherty, E. M.; Hannick, S. M.; Codavoci, L.; Henry, R. F.; Green, B. E.; Spanton, S. G.; Norbeck, D. W. J. Org. Chem. 1992, $57,5692$.

2. Wang, G. T.; Li, S.; Wideburg, N.; Krafft, G. A.; Kempf, D. J. J. Med. Chem. 1995, 38, 2995.

3. Chen, P.; Cheng, P. T. W.; Alam, M.; Beyer, B. D.; Bisacchi, G. S.; Dejneka, T.; Evans, A. J.; Greytok, J. A.; Hermsmeier, M. A.; Humphreys, W. G.; Jacobs, G. A.; Kocy, O.; Lin, P.; Lis, K. A.; Marella, M. A.; Ryono, D. E.; Sheaffer, A. K.; Spergel, S. H.; Sun, C.; Tino, J. A.; Vite G.; Colonno, R. J.; Zahler, R.; Barrish, J. C. J. Med. Chem. 1996, 39, 1991.

4. Alcon, M.; Poch, M.; Moyano, A.; Pericas, M. A.; Riera, A. Tetrahedron: Asymmetry 1997, 8, 2967.

5. Alexander, C. W.; Liotta, D. C. Tetrahedron Lett. 1996, 37, 1961.

6. Beaulieu, P. L.; Gillard, J.; Bailey, M.; Beaulieu, C.; Duceppe, J.; Lavallée, P.; Wernic, D. J. Org. Chem. 1999, 64, 6622.

7. Bergmeier, S. C. Tetrahedron 2000, 56, 2561.

8. Hetényi, A.; Szakonyi, Z.; Klika, K. D.; Pihlaja, K.; Fülöp, F. J. Org. Chem. 2003, 68, 2175.

9. Flanagan, S. P.; Guiry, P. J. J. Organomet. Chem. 2006, 691, 2125 and references cited therein.

10. Lait, S. M.; Parvez, M.; Keay, B. A. Tetrahedron: Asymmetry 2004, 15, 155.

11. Mino, T.; Hata, S.; Ohtaka, K.; Sakamoto, M.; Fujita, T. Tetrahedron Lett. 2001, 42, 4837.

12. Tang, W.; Zhang, X. Chem. Rev. 2003, 103, 3029 and references cited therein.

13. Palomo, C.; Oiarbide, M.; Laso, A. Eur. J. Org. Chem. 2007, 16, 2561.

14. Mishra, R. K.; Coates, C. M.; Revell, K. D.; Turos, E. Org. Lett. 2007, 9, 575.

15. Grajewska, A.; Rozwadowska, M. D. Tetrahedron: Asymmetry 2007, 18, 803.

16. Szakonyi, Z.; Martinek, T. A.; Hetényi, A.; Fülöp, F. Tetrahedron: Asymmetry 2000, 11, 4571.

17. Szakonyi, Z.; Fülöp, F. Arkivoc 2003, 14, 225.

18. Gyónfalvi, S.; Szakonyi, Z.; Fülöp, F. Tetrahedron: Asymmetry 2003, 14, 3965.

19. Szakonyi, Z.; Balázs, Á.; Martinek, T. A.; Fülöp, F. Tetrahedron: Asymmetry, 2006, 17, 199.

20. Szakonyi, Z.; Hetényi, A.; Fülöp, F. Tetrahedron in press, doi:10.1016/j.tet.2007.07.065.

21. Lavallée, P.; Bouthillier, G. J. Org. Chem. 1986, 51, 1362.

22. Lakshmi, R.; Bateman, T. D.; McIntosh, M. C. J. Org. Chem. 2005, 70, 5313.

23. Scheidl, F. Synthesis 1982, 728.

24. Tahtinen, P.; Sinkkonen, J.; Klika, K. D.; Nieminen, V.; Stájer, G.; Szakonyi, Z.; Fülöp, F.; Pihlaja, K. Chirality 2002, 14, 187.

25. Frisch, M. J.; Trucks, G. W.; Schlegel, H. B.; Scuseria, G. E.; Robb, M. A.; Cheeseman, J. R.; Montgomery, J. A., Jr.; Vreven, T.; Kudin, K. N.; Burant, J. C.; Millam, J. M.; Iyengar, S. S.; Tomasi, J.; Barone, V.; Mennucci, B.; Cossi, M.; Scalmani, G.; Rega, N.; Petersson, G. A.; Nakatsuji, H.; Hada, M.; Ehara, M.; Toyota, K.; Fukuda, R.; Hasegawa, J.; Ishida, 
M.; Nakajima, T.; Honda, Y.; Kitao, O.; Nakai, H.; Klene, M.; Li, X.; Knox, J. E.; Hratchian, H. P.; Cross, J. B.; Adamo, C.; Jaramillo, J.; Gomperts, R.; Stratmann, R. E.; Yazyev, O.; Austin, A. J.; Cammi, R.; Pomelli, C.; Ochterski, J. W.; Ayala, P. Y.; Morokuma, K.; Voth, G. A.; Salvador, P.; Dannenberg, J. J.; Zakrzewski, V. G.; Dapprich, S.; Daniels, A. D.; Strain, M. C.; Farkas, O.; Malick, D. K.; Rabuck, A. D.; Raghavachari, K.; Foresman, J. B.; Ortiz, J. V.; Cui, Q.; Baboul, A. G.; Clifford, S.; Cioslowski, J.; Stefanov, B. B.; Liu, G.; Liashenko, A.; Piskorz, P.; Komaromi, I.; Martin, R. L.; Fox, D. J.; Keith, T.; Al-Laham, M. A.; Peng, C. Y.; Nanayakkara, A.; Challacombe, M.; Gill, P. M. W.; Johnson, B.; Chen, W.; Wong, M. W.; Gonzalez, C.; Pople, J. A. Gaussian 03, Revision A.1; Gaussian, Inc.: Pittsburgh, PA, 2003; http:/www.gaussian.com/. 